is hoped that, at least, the complement-fixation test has been proved to be of real value in the diagnosis of gonorrhoea.

The most striking points are :-

1. The early stage at which a positive result may be obtained.

2. The relatively high percentage of positives in undoubted cases of active gonorrhoea (average $86 \cdot 5$ per cent.).

3. The very low incidence of "false positives" only 3 in 1000 . In none of these cases could gonorrhœea be absolutely excluded, and the cases were only included in category 4 because the medical officers in charge of the cases marked them " nonvenereal"; perhaps they had not much faith in the complement-fixation test!

4. A "doubtful" test is at least suspicious, and should be repeated. It should also stimulate a further careful examination.

The test is capable, no doubt, of being rendered much more delicate; in view of the large number of doubtful results obtained, attempts are being made to accomplish this. I have not been able to come to any definite conclusion as to how long a serum may remain positive after the patient is cured. Tests of cure are laborious and very unsatisfactory, and few patients can be induced to return for examination after they think they are free from their gonorrhoea; the " man in the street" treats gonorrhoa much more lightly than he does syphilis. It is hoped, nevertheless, to repeat many of the above tests over a lengthy period in order to arrive at some definite conclusion. It has been found (particularly in cases showing complications) that a positive reaction frequently persists for 12 months or more after apparent cure, and many of the " doubtful" results were obtained in cases which had showed no clinical signs for an even longer period ; these represent many of the "doubtful" results in category 3 . This appears to be contrary to the findings of most workers.

In conclusion, my thanks are due to Colonel L. W. Harrison, D.S.O., director of the venereal diseases clinic at St. Thomas's Hospital, for permission to publish these notes, and to the medical officers of the clinic for providing me with the material necessary for this investigation; also to the clerks in the male clinic, and to the ladies in the lady almoner's department, for searching for and re-filing a large number of cards on a great many occasions.

Referenees.-1. Jour. R.A.M.C., February, 1914. 2. Proc. Roy. Soc. Med., December, 1912, 3. THE IAANCET, 1919, i., Com., Spec. Rep. Series, No. 19 .

\section{BASAL FIBROSIS IN CHILDREN.}

By G. T. HEBERT, M.D. OXF., M.R.C.P. LoND., PHYSICIAN IN CHARGE OF TUBFRCULOSIS DEPARTMENT, ST. THOMAS'S HOSPITAI, LONDON.

IN September, 1920, in an article entitled Basal Pneumonic Residues in Children, ${ }^{1}$ Dr. Walker Overend showed that these residues appear in his radiograms as opaque areas, often with a sharp edge and sometimes with accompanying dilatation of bronchi. The term "basal pneumonic residues" presumably includes that not uncommon affection of children which, in the absence of a satisfactory name, is called fibroid disease, non-tuberculous pulmonary fibrosis, chronic interstitial pneumonia, or sometimes bronchiolectasis, and which, on account of the well-marked signs discoverable on examination of the chest, is often mistaken for tuberculosis. It is an undoubted fact that some cases of this group show on radiological examination an increased translucency, instead of an opacity, in the affected area.
This phenomenon was undoubtedly present in six of the cases of basal fibrosis, probably non-tuberculous in origin, seen in the tuberculosis department of St. Thomas's Hospital during the last year. All gave a similar history and presented similar physical signs.

The following is a typical case :-

H. A., aged 11, was sent to hospital in November, 1918, by a practitioner who had found abnormal signs in his chest. Measles at the age of 3 months had been followed by broncho-pneumonia, and every subsequent winter he had had a cough, sometimes with a little expectoration. He was said to be short of breath, but had a good appetite and appeared to be bright and well. Diminished expansion was noticed on the right side of his chest especially at the lower part, where there were also impairment of percussion note, deficient air entry, and numerous coarse crepitations. The left lung was apparently normal. For a year he remained under observation, keeping well, and attending school, but was then sent to a sanatorium from which he has just returned after a stay of over two years. All sputum examinations have been negative for tubercle bacilli; he has been apyrexial, has gained weight steadily, and has been comparatively free from symptoms. His signs are now the same as they were three years ago, with the addition of slight hollowing of the chest wall on the right side and displacement of the heart to the right.

A radiogram shows (a) displacement of the heart half an inch to the right; $(b)$ increased translucency of the lower half of the right pulmonary field; (c) absence of the smaller shadows of the bronchial tree and replacement of the larger by a fine linear marking; and (d) shadows suggesting some increase of fibrous tissue round the bronchi at the root of the lung with slight dilatation of the bronchioles at this edge of the translucent area.

\section{Remarks.}

These unusual appearances in the radiogram were not due to faulty technique, for the tube had been centred correctly so as to give an even illumination of the chest; nor was absence of bronchial shadows due to voluntary or involuntary movement during exposure, for all of the shadows present were sharply defined. Though the converse was not always true, all of the cases showing the translucent area showed displacement of the heart to that side, and presumably were definite cases of fibrosis. But fibrous tissue is generally opaque. A densely fibrotic tuberculous lung, for instance, shows a general opacity with comparatively little detail ; and a less severe fibrosis, as seen in a series of radiograms from a mild case of tuberculosis, may show the replacement of a hazy area, representing active disease-but due, perhaps, largely to congestion-by linear shadows which become quite definite after six months.

The fact that in the cases of non-tuberculous fibrosis under consideration the fibrous tissue does not cast the usual shadow is difficult to explain. It is improbable that the fibrous tissue is in an unformed, perhaps a gelatinous, state, for the length of history in these cases may be ten years or more. It is more probable that the normal shadows of fibrous tissue are not seen because the surrounding lung parenchyma is avascular, emphysematous, cdematous, or in some other state which renders it unusually translucent. Of the possibilities mentioned, cedema is the most probable from the pathological standpoint. It may be objected that an cedematous area of lung tissue should be opaque to $X$ rays rather than translucent. Free fluid in the chest is, of course, opaque, but it does not follow that fluid in the tissue of the lung, with slight exudation into the air-containing structures, produces the same appearance. From the clinical standpoint the explanation of the crepitations is not clear. It is very unlikely that in the majority of cases they are caused by secretion. Some of the finer sounds may be produced in collapsed areas, but the remainder, being medium-sized and typically moist, suggest the presence of fluid in structures larger than the alveoli. It is possible, therefore, that in some cases of basal fibrosis the interstitial tissue becomes cedematous and then shows the $X$ ray appearance noted above. But whatever its explanation, this translucency is, it is claimed, an important piece of evidence in the differentiation of non-tuberculous from tuberculous fibrosis of the lungs, for in the latter it does not appear to occur. 


\section{THE SYSTEMATIC USE OF POISSON'S FORMULA.}

BY G. DE M. RUDOLF, M.R.C.S. ENG., D.P.H., SECOND CASUALTY OFFICER, KING'S COLXEGE HOSPITAL.

THe value of statistics depends to a great extent upon the use that can be made of the figures by succeeding investigators. If the latter, proceeding along the same lines as the original inquirer, are able correctly to compare their own figures with those of the first author, the statistics are of great value, but if the two sets of figures are not strictly comparable the statistics are of less value. In a great number of statistics the figures are not reduced to a reliable common basis. The two following instances illustrate this point.

The late Sir William Osler ${ }^{1}$ found that, in 100 autopsies of cases of lobar pneumonia, both lungs were affected in 17 patients-that is, 17 per cent. Again, Dr. Aldren Turner ${ }^{2}$ records that in 200 autopsies on intracranial tumours, 96 , or 48 per cent., of the growths were found to be gliomata. Now, other investigators proceeding along the same lines as
If $\mathrm{M}=$ total number of observations recorded; $\mathbf{m}=$ number of observations in one group

$\mathbf{n}=$ number of observations in the other group. and $m+n=M$,

then Poisson's rule states that the maximum varia. tion possible $=\sqrt{\frac{2 m n}{M^{3}}}$, and the variation per cent. $=100\left(2 . \sqrt{\frac{2 \mathrm{mn}}{\mathrm{M}^{3}}}\right)$

An example will show how the formula can be applied. Dr. G. F. Still ${ }^{4}$ states that in 383 cases of chorea, 183 , or 47.48 per cent., had concurrent or previous articular rheumatism. In this instance therefore $\mathrm{M}=383 ; \mathrm{m}=183$ (or 200); $\mathrm{n}=200$ (or 183) ;

$$
\begin{aligned}
\therefore \quad \sqrt{\frac{2 \mathrm{mn}}{\mathrm{M}^{3}}}=2 . \sqrt{\frac{2 \times 183 \times 200}{383^{3}}} & =0.07218 \\
& =7.218 \text { per cent. }
\end{aligned}
$$

Consequently, another observer recording cases of chorea with articular rheumatism might expect to find that his results differed from those of Dr. Still by 7.218 per cent. : thus his results would lie any. where between 47.78 per cent. +7.218 per cent. and 47.78 per cent. $-7 \cdot 218$ per cent.; that is, between

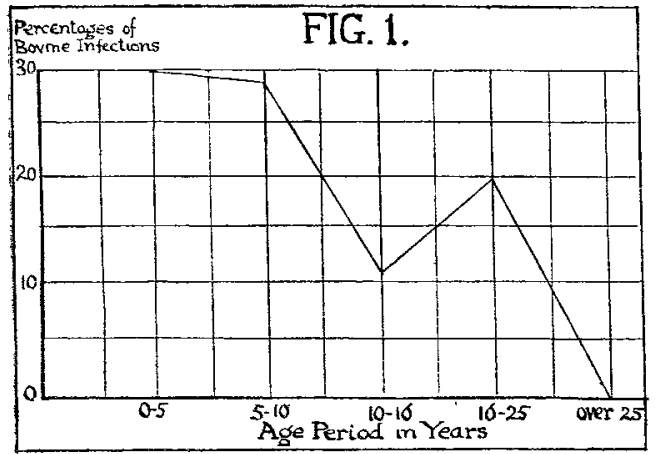

Results obtained by Eastwood and Griffith.

either Sir William Osler or Dr. Aldren Turner would expect to obtain approximately the same results in the respective observations as did these two inquirers. The investigator inquiring into the percentage occurrence of gliomata

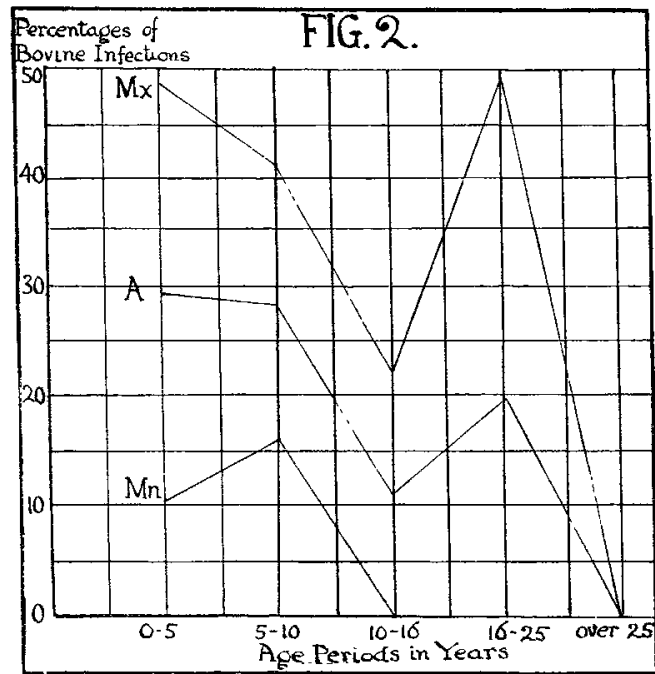

A represents graph of original observations. Mx the maximum percentage that could be found by other investigators. Mn the minimum percentage.

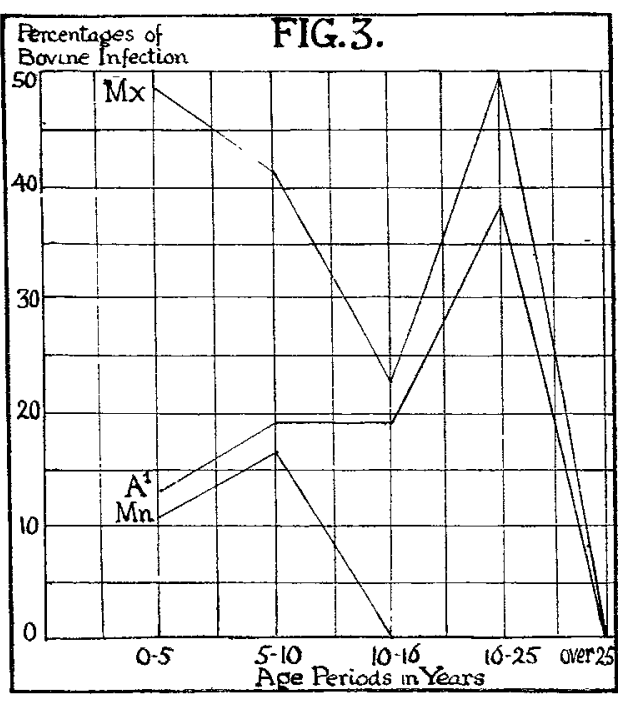

${ }^{1}$ represents results apparently differing from those drawn in Fig. 1, but actually confirming them. is, however, more likely to obtain results closely approaching those observed by Dr. Aldren Turner than another investigator inquiring into the condition of the lungs in pneumonia likely to approach the results of Sir William Osler ; Osler's 17 per cent. was based upon a study of 100 cases, whereas Aldren Turner's 48 per cent. Was based upon a study of 200 cases. Consequently, although these results have been expressed in a similar way-i.e. as the number of certain cases found in 100 autopsies of the particular disease--Aldren 'Turner's results are of more value than those of Sir William Osler. It follows, therefore, that the expression of a series of results as a percentage is of little value unless the total number of cases observed is also stated.

\section{Method of Estimating Maximum Possible} Variation.

There is, however, a well-known method which, when applied to a series of observations, shows the maximum variation possible which could be found in any other series of similar observations. This method depends upon Poisson's rule. ${ }^{3}$ If, in a series of observations, of which one part shows one possibility and the other part, a second possibility, and the total number of observations in the two parts is equal to the total number of observations in the whole series, the following formula be applied, the maximum possible variation of any subsequent series can be determined, provided that the second series was as great as or greater than the first series.

54.998 per cent. and 40.562 per cent. Of course, the second observer would only expect to find his results falling between these limits if the number of cases in his series was as great as that in Dr. Still's series. If this were the case, and his results lay between 54.998 per cent. and $40 \cdot 562$ per cent., then his work would be in accordance with that of Dr. Still, although his figures might vary from those obtained by Dr. Still by 7.218 per cent.

The maximum variation possible does not depend entirely upon the total number of observations made, for in the series of observations quoted above, Dr. Still found that two cases--that is, 0.5222 per cent.had rheumatic nodules without cardiac bruits on joint symptoms. If the formula be applied in this case it will be found that the maximum variation possible is 1.041 per cent. Therefore in another series of cases of chorea the number of patients found with rheumatic nodules, but without cardiac bruits or joint symptoms, would lie between 1.563 per cent. and 0.519 per cent.

\section{A Method of Plotting Graphs in Conjunction with} Poisson's Rule.

The object of this article can now be explained. It is the description of a method, which I believe to be new, of plotting graphs of statistics in conjunction with Poisson's rule, whereby the graph is made of greater value than if it were plotted in the usual way. An example will show the method which I sugrest.

Hewlett ${ }^{5}$ quotes the work of Eastwood and Griffith who, investigating the characteristics of the tubercle 